\title{
Connectomics in high throughput
}

\author{
BRICseq provides access to brain-wide connectivity and can be combined with functional and transcriptomic \\ studies for a comprehensive view of neuronal circuitry.
}

K nowledge of how brain regions are connected to each other is key to understanding how their connectivity drives behavior. However, assessing connectivity with whole-brain coverage has typically been a laborious process that involved injecting viral tracers and imaging their distributions in countless brains. Anthony Zador from the Cold Spring Harbor Laboratory in Cold Spring Harbor, New York, and his colleagues have built on their previously published MAPseq technology and established a high-throughput approach for connectome mapping, called brain-wide individual animal connectome sequencing or BRICseq.

Zador says that he was thinking about a high-throughput connectomics approach more than ten years ago, which eventually led to the idea of barcoding neurons. An important step towards the goal was the work of graduate student Ian Peikon, "who pursued the idea that we could express RNA barcodes and then drag them out to synapses," says Zador. In close work with another graduate student, Justus Kebschull, MAPseq was established.

In MAPseq, a brain region of interest is injected with a Sindbis virus library that encodes RNA barcodes as well as a peptide that targets the barcodes to projections. One to two days after infection, the brain is removed and subdivided into slices, which are then analyzed by sequencing. This approach allows determination of innervation patterns. For example, if a neuron targets multiple brain regions, the same barcode will be present in these different brain regions.

The newly published BRICseq scales this approach up. This project was spearheaded by postdoc Longwen Huang, who "wanted to scale up the projectome tracing to whole-brain tracing," according to Zador. The goal was to tile the cortex with hundreds of injections and then generate a comprehensive region-to-region connectome by dividing the brain into hundreds of cubelets and sequencing the barcodes present in these cubelets. The team had early indications that the methodology could work when injecting at multiple sites in the brain. However, such a scenario is complicated by the fact that barcodes appear at both the injection and the projection
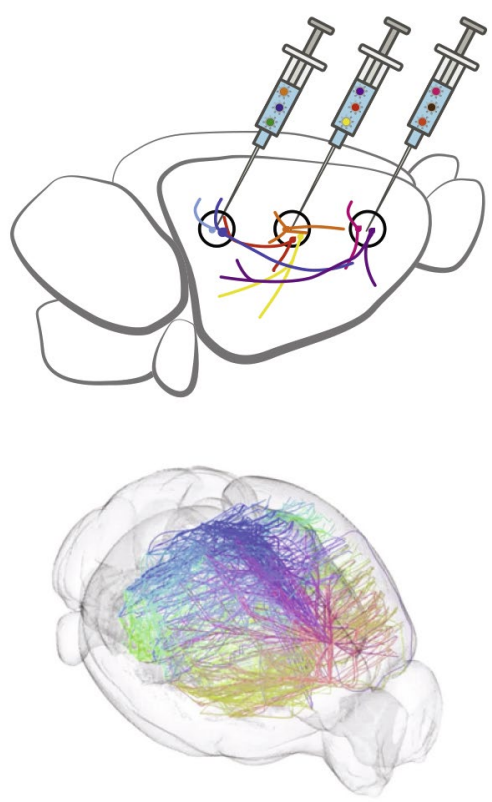

Schematic of BRICseq (top) and connectivity map in a mouse brain (bottom). Reprinted with permission from Cell 182, 177-188, Elsevier.

sites. "You don't know where the barcodes were injected, which is the source or which is the target," explains Zador. While the team initially contemplated adding a second barcode to encode the injection site, such an approach would not be conducive to high throughput, as hundreds of libraries would have to be generated, one for each injection site. Luckily, "it turns out that the amount of barcode is proportional to the amount of cell volume," says Zador. In other words, high levels of a barcode correlate with the presence of the cell body of the infected neuron.

BRICseq appears to be a straightforward extension of MAPseq, but "that turned out to actually be more challenging than we originally thought," says Zador. For example, while formation of chimeras between barcodes was a negligible problem in MAPseq, it was troublesome when scaling up to BRICseq. Another challenge was making sure that the more than 300 cubelets that each brain was divided into were properly aligned to the original brain. Now that
BRICseq is established, "we're able to do in a couple of weeks, in terms of throughput, what using standard single-neuron tracing technique takes years," says Zador, although there is still room for further optimization.

While high throughput is one advantage of BRICseq, the methodology is also robust. "You never want to do just one brain, but it turns out that the interbrain variability is surprisingly low," says Zador. The main sources of variability are the injection and sectioning procedures, while the barcode sequencing does not contribute much to variability.

Making use of the robustness of BRICseq-derived connectomes, the researchers compared these connectomes with functional connectomes established by widefield calcium imaging during an auditory decision-making task. They observed strong correlation between the two types of connectivity maps. Similarly, they observed correlations between gene expression data and BRICseq data, suggesting that gene expression data can be used to predict connectivity. Furthermore, the researchers used the approach to compare connectomes between C57BL/6J mice and a mutant mouse strain that lacks the corpus callosum. As expected, the researchers found that the mutant mice were devoid of commissural cortical connections.

Zador is excited about the possibilities that BRICseq and related methods open up for his research in auditory decision-making processes. He plans to combine sequencing-based connectomics approaches with calcium imaging and transcriptomics in mice that carried out a decision-making task. "My hope is that putting those three sources of information together will really be able to provide an understanding of the contributions of the incredibly heterogeneous populations of neurons in the cortex," says Zador.

Nina Vogt

Published online: 1 September 2020 https://doi.org/10.1038/s41592-020-0948-z

Research paper

Huang, L. et al. BRICseq bridges brain-wide interregional connectivity to neural activity and gene expression in single animals. Cell 182, 177-188 (2020). 